\title{
Surgical outcomes of stage IV non-small cell lung cancer: a single-center experience
}

\author{
Chenxi Zhang", Lei Wang", Weimiao Li", Zhao Huang, Wenhao Liu, Peilong Bao, Yuanyang Lai, \\ Yong Han, Xiaofei Li, Jinbo Zhao
}

Department of Thoracic Surgery, Tangdu Hospital, Air Force Medical University (The Fourth Military Medical University), Xi'an 710038, China Contributions: (I) Conception and design: C Zhang, L Wang, W Li, X Li, J Zhao; (II) Administrative support: X Li, J Zhao; (III) Provision of study materials or patients: P Bao, Y Lai, Y Han; (IV) Collection and assembly of data: C Zhang, L Wang, W Li, Z Huang, W Liu; (V) Data analysis and interpretation: C Zhang, L Wang, W Li, X Li, J Zhao; (VI) Manuscript writing: All authors; (VII) Final approval of manuscript: All authors.

\#These authors contribute equally to this work.

Correspondence to: Jinbo Zhao. Department of Thoracic Surgery, Tangdu Hospital, Air Force Medical University (The Fourth Military Medical University), No. 569, Xinsi Road, Xi'an 710038, China. Email: zhaojinbo@aliyun.com; Xiaofei Li. Department of Thoracic Surgery, Tangdu Hospital, Air Force Medical University (The Fourth Military Medical University), No. 569, Xinsi Road, Xi'an 710038, China. Email: 1xfchest@fmmu.edu.cn.

Background: Increasing evidence has shown the effectiveness of surgery for stage IV non-small cell lung cancer (NSCLC). Present study aims to summarize the experience of our institution in dealing with advanced NSCLC in the context of multimodality therapy including lung surgery.

Methods: Patients underwent surgical resection for stage IV NSCLC diagnosed before or during surgery from January 2014 to June 2017 at Tangdu Hospital were included in this study.

Results: There were 88 stage IV NSCLC patients enrolled in this study. Among them, 35 patients with pleural metastases, 18 with brain oligometastases, 25 with extra-brain oligometastases and 10 with multiple metastatic sites or organs. For primary lung tumor, almost all (86/88) were resected with R0. For metastatic lesions, 53 patients received curative local treatment and 9 patients with partial treatment. There were 62 patients received adjuvant treatment, 10 patients received no adjuvant treatment and 16 patients with missing data of adjuvant treatment. The median overall survival of patients was 31.72 months. The estimated 3-year OS was $42.2 \%$. Patients with pleural metastases and brain oligometastases got better outcomes than the ones with extra-brain oligometastases and multiple metastases $(\mathrm{P}<0.001)$. Patients with adjuvant epidermal growth factor receptor (EGFR) tyrosine kinase inhibitor (TKI) treatment had significantly better OS compared with those with adjuvant chemotherapy treatment $(\mathrm{P}=0.015)$. Besides, age $<60$ and $\mathrm{cT} 1-2$ were also associated with better survival.

Conclusions: Surgery may be a considerable choice for stage IV NSCLC in the context of multimodality therapy.

Keywords: Non-small cell lung cancer (NSCLC); surgery; multimodality therapy; targeted therapy; overall survival (OS)

Submitted Apr 26, 2019. Accepted for publication Sep 03, 2019.

doi: $10.21037 /$ jtd.2019.11.30

View this article at: http://dx.doi.org/10.21037/jtd.2019.11.30 


\section{Introduction}

Lung cancer remains the leading cause of cancer-related death worldwide, with the estimated annual mortality of 1.8 million in 2018 (1). As the major histologic type, non-small cell lung cancer (NSCLC) accounts for $85 \%$ of all cases. Almost half of NSCLC patients (46.8-61.2\%) have been reported to have stage IV disease at their initial diagnosis $(2,3)$. Patients with this advanced-stage disease are generally assumed to be incurable and therefore are treated with palliative intent. Systemic chemotherapy is the principle therapeutic strategy for stage IV NSCLC patients (4). With the remarkable development of targeted therapy, survival of stage IV NSCLC has been significantly improved $(5,6)$. Nevertheless, few of these patients survived more than 5 years.

Increasing evidence has suggested the survival benefits of surgical resection in highly selected advanced patients. Surgery for some selected stage IV NSCLC prolonged survival of patients (7-9). A national analysis of long-term surgical outcomes from America by Yang and colleagues showed a better 5 -year OS of $25 \%$ in patients with cT1-2, N0-1, M1 or cT3, N0, M1 disease when compared with non-surgical interventions (5.8\% for chemoradiation, $5.9 \%$ for chemotherapy, and $3.2 \%$ for radiotherapy) (10). All these findings support the advantages of surgical resection for specific patients with this end-stage disease.

However, there is still limited evidence on selection of stage IV NSCLC for surgical treatment. The present study was therefore carried out to summarize the experiences of the surgical outcomes of stage IV NSCLC in the context of multimodality therapy. A single-center experience in this study may help to better understand the prognosis of multimodality treated stage IV NSCLC in the era of precision medicine.

\section{Methods}

\section{Patients}

We retrospectively reviewed the medical records of 4,015 consecutive NSCLC patients who underwent surgical resection during January 2014 to June 2017 at the Department of Thoracic Surgery, Tangdu Hospital, China. A total of 112 patients were documented to have stage IV NSCLC preoperatively or during surgery. Of these, 18 patients who underwent exploratory procedures were excluded. We also excluded those who had second primary malignancy in 5 years $(n=3)$ or underwent second surgery for recurrent diseases $(n=3)$. This resulted in 88 patients eligible for final analysis. All patients were reclassified according to the 8th TNM staging system. Chest and upper-abdominal high-resolution computed tomography, bronchoscopic biopsy, brain magnetic resonance imaging and bone scintigraphy were routinely performed for most patients. Positron emission tomography and computer tomography (PET/CT) was used at the discretion of surgeons according to the patient's condition. The study protocol was approved by the institutional review board of Tangdu Hospital (No. 201712-25). Informed consent was waived for this retrospective observational study.

\section{Data collection}

Data on patient characteristics, treatment and clinical outcomes were retrieved from medical records based on the LinkDoc database. Clinical characteristics analyzed were age at surgery, sex, smoking history, clinical $\mathrm{T}$ and $\mathrm{N}$ stage, tumor laterality, histology and metastases, as well as molecular test performed. Data on medical comorbidity was also collected and Charlson comorbidity index was calculated for each patient. Treatment-related variables included pulmonary resection (sublobar resection, lobectomy, or pneumonectomy) and margin (R0 or R1/2), adjuvant therapy (chemotherapy and/or targeted therapy), local treatment for metastases (Curative treatments: complete resection or stereotactic body radiotherapy or radiofrequency ablation; Partial treatment: palliative surgery for metastases or partial resection of multiple metastases), perioperative complication and mortality. Follow-up information was obtained from medical records or by telephone interview.

\section{Statistical analysis}

The primary endpoint was OS of patients that was calculated from the date of surgery to the date of death or last contact. Survival curve was developed by Kaplan-Meier method and subgroup analysis was compared by Logrank test. Multivariate Cox proportional hazards model was established to estimate the hazard ratio (HR) and $95 \%$ confidence interval (95\% CI). Variables in univariate analysis with a $\mathrm{P}$ value of $<0.05$ were entered into the multivariate model. A P value of $<0.05$ was considered statistically significant. All statistical analysis was carried out with SPSS 13.0 (SPSS Inc., Chicago, IL, USA). 
Table 1 Clinical characteristics of surgically resected stage IV patients

\begin{tabular}{|c|c|}
\hline Variable & Total $(n=88)$ \\
\hline \multicolumn{2}{|l|}{ Sex, n (\%) } \\
\hline Male & $54(61.4)$ \\
\hline Female & $34(38.6)$ \\
\hline \multicolumn{2}{|c|}{ Age at surgery, n (\%) } \\
\hline$<60$ years & $53(60.2)$ \\
\hline$\geq 60$ years & $35(39.8)$ \\
\hline \multicolumn{2}{|c|}{ Smoking history, n (\%) } \\
\hline Yes & $37(42.0)$ \\
\hline No & $51(58.0)$ \\
\hline \multicolumn{2}{|c|}{ CCl score, n (\%) } \\
\hline $0-1$ & $84(95.5)$ \\
\hline $2-3$ & $4(4.5)$ \\
\hline \multicolumn{2}{|c|}{ cT stage, n (\%) } \\
\hline T1 & $22(25.0)$ \\
\hline T2 & $43(48.9)$ \\
\hline T3 & $10(11.4)$ \\
\hline $\mathrm{T} 4$ & $13(14.8)$ \\
\hline \multicolumn{2}{|c|}{ pT stage, n (\%) } \\
\hline T1 & $13(14.8)$ \\
\hline $\mathrm{T} 2$ & $26(29.6)$ \\
\hline T3 & $14(15.9)$ \\
\hline $\mathrm{T} 4$ & $35(39.8)$ \\
\hline \multicolumn{2}{|c|}{ cN stage, n (\%) } \\
\hline No & $61(69.3)$ \\
\hline $\mathrm{N} 1$ & $4(4.5)$ \\
\hline N2 & $20(22.7)$ \\
\hline N3 & $3(3.4)$ \\
\hline \multicolumn{2}{|c|}{ pN stage, n (\%) } \\
\hline No & $24(27.3)$ \\
\hline $\mathrm{N} 1$ & $10(11.4)$ \\
\hline N2 & $51(58.0)$ \\
\hline N3 & $3(3.4)$ \\
\hline \multicolumn{2}{|c|}{ Tumor location, n (\%) } \\
\hline Left lung & $39(44.3)$ \\
\hline Right lung & $49(55.7)$ \\
\hline
\end{tabular}

Table 1 (continued)
Table 1 (continued)

\begin{tabular}{lc}
\hline Variable & Total $(\mathrm{n}=88)$ \\
\hline Surgical approach, $\mathrm{n}(\%)$ & $35(39.8)$ \\
VATs or robots & $53(60.2)$ \\
Thoracotomy or conversion & \\
Histology, $\mathrm{n}(\%)$ & $68(77.3)$ \\
Adenocarcinoma & $11(12.5)$ \\
Squamous cell carcinoma & $9(10.2)$ \\
Others ${ }^{\dagger}$ & \\
Metastatic sites, $\mathrm{n}(\%)$ & $35(39.8)$ \\
Pleural metastases & $18(20.5)$ \\
1-3 brain metastases & $25(28.4)$ \\
1-3 extra-brain metastases & $10(11.4)$ \\
Multiple metastases & \\
Mutation test, $\mathrm{n}(\%)$ & $38(43.2)$ \\
EGFR/ALK/ROS1 positive ${ }^{\ddagger}$ & $40(45.5)$ \\
EGFR/ALK/ROS1 negative & $10(11.4)$ \\
Unknown & \\
\hline
\end{tabular}

t: 6 adenosquamous carcinomas, 2 large cell carcinomas, 1 polymorphic cell carcinomas; ‘: 36 cases with EGFR mutation, 1 case with ALK rearrangements and 1 case with ROS1 rearrangements. $\mathrm{CCl}$, Charlson comorbidity index; EGFR, epidermal growth factor receptor; ALK, anaplastic lymphoma kinase.

\section{Results}

\section{Patients characteristics}

Among 88 surgical stage IV patients, 54 (61.4\%) were male. The median age was 57.4 years at the time of surgery. The clinical characteristics of patients are shown in Table 1. All patients were with performance status $0-1$ point. Most patients had no comorbidity (92.1\%). A histologic analysis showed $68(77.3 \%)$ adenocarcinoma, $11(12.5 \%)$ squamous cell carcinoma and $9(10.2 \%)$ other types (6 adenosquamous carcinomas, 2 large cell carcinomas and 1 polymorphic cell carcinomas). There were 35 patients with pleural metastases alone, 14 of which were diagnosed before surgery. Eighteen patients with 1-3 brain metastases and 25 patients with 1-3 extra-brain metastases. The others were 10 patients who had multiple metastases (more than 3 metastases in single organ or metastases in multiple organs). Among these, 3 patients were diagnosed with pleural dissemination before 
Table 2 Primary lung surgery combined adjuvant treatment and postoperative outcomes

\begin{tabular}{|c|c|}
\hline Variable & Total $(n=88)$ \\
\hline \multicolumn{2}{|l|}{ Pulmonary resection, n (\%) } \\
\hline Sublobar resection & $5(5.7)$ \\
\hline Lobectomy & $60(68.2)$ \\
\hline Pneumonectomy & $23(26.1)$ \\
\hline \multicolumn{2}{|l|}{ Pulmonary margin, $\mathrm{n}(\%)$} \\
\hline Ro & $86(97.7)$ \\
\hline $\mathrm{R} 1$ & $2(2.3)$ \\
\hline \multicolumn{2}{|l|}{ Adjuvant therapy, n (\%) } \\
\hline Chemotherapy & $31(35.2)$ \\
\hline $\mathrm{TKI}$ or $\mathrm{TKI}+$ chemotherapy $^{\dagger}$ & $31(35.2)$ \\
\hline No treatment & $10(11.4)$ \\
\hline Unknown $^{\ddagger}$ & $16(18.2)$ \\
\hline Postoperative complications, n (\%) & $8(9.1)$ \\
\hline Respiratory failure & $2(2.3)$ \\
\hline Bronchial fistula & $2(2.3)$ \\
\hline Atelectasis + respiratory failure & $1(1.1)$ \\
\hline Air leakage & $1(1.1)$ \\
\hline Chylothorax & $1(1.1)$ \\
\hline Pulmonary embolism & $1(1.1)$ \\
\hline Postoperative mortality at 30 days, $\mathrm{n}(\%)$ & $1(1.1)$ \\
\hline
\end{tabular}

${ }^{\dagger}, 31$ cases with EGFR mutant; ${ }^{\ddagger}$, no adjuvant therapy data of 5 case with EGFR mutation, 1 case with ALK rearrangements and 1 case with ROS1 rearrangements. TKI, tyrosine-kinase inhibitor; SCC, squamous cell carcinoma.

surgery, and continued to develop distant metastases in three months after surgery. They were also included in this study because their short-term metastases were considered as synchronous metastases. Mutation test was performed on 78 (88.6\%) patients. Of these, 38 (43.2\%) harbored EGFR or ALK or c-ros oncogene 1 receptor tyrosine kinase (ROS1) mutations.

\section{Treatment strategy}

The selection of surgical treatment patients

All eligible patients selected for surgery were in good generally conditions and discussed by multidisciplinary team before surgery while metastases could be potentially treated with local treatment.

The surgical treatments for primary lung tumors, the adjuvant treatments and postoperative outcomes

All patients underwent surgical resection for primary tumors. As show in Table 2, 60 (68.2\%) patients were managed by lobectomy, 23 (26.1\%) by pneumonectomy and $5(5.7 \%)$ by sublobar resection (4 wedge resection and 1 segmentectomy). Almost all (97.7\%) patients achieved R0 pulmonary resection (primary lung lesion, not including pleural dissemination).

Sixty-two $(70.5 \%)$ patients were treated by adjuvant therapies, $10(11.4 \%)$ patients received no adjuvant therapy and $16(18.2 \%)$ patients with unavailable adjuvant treatment data. Of these with available adjuvant treatment data, 31 were treated with chemotherapy and the other 31 were with EGFR tyrosine-kinase inhibitor (TKI) or EGFR-TKI plus chemotherapy. Adjuvant treatment data of 3 patients with EGFR, 1 patient with ALK and 1 patient with ROS1 rearrangement were unknown. Two patients with EGFR mutation were lost follow-up. No patients enrolled in this study received induction therapy.

Eight patients had postoperative complications. One with pulmonary embolism died 30 days after pneumonectomy.

\section{The treatments for metastases}

There were 35 patients with pleural metastases alone. For the treatments of the pleural metastases, 23 patients received curative treatment (complete resection), 4 patients received partial treatment (partial resection of multiple pleural metastases), and 8 patients received no treatment (Table S1).

All 18 brain oligometastatic patients received curative treatment for brain lesions, including surgery and SRS with or without combination of whole brain radiotherapy (WBRT). Local treatment for brain metastases were routinely performed before lung resection (Table S2).

There were 25 patients with extra-brain oligometastases. Curative treatments were performed on 10 patients, partial treatment was performed on 2 patients and no treatment on the rest 13 patients (Table S3).

Totally 10 patients were with multiple metastases or metastases in multiple organs. Five patients were with pleural plus distant organ metastases. Of these, 2 patients received curative treatment and 3 patients received partial treatment. No local therapies were performed on the 5 patients with multiple distant organ metastases (Table S4).

Adjuvant EGFR-TKI therapy for patients with different 


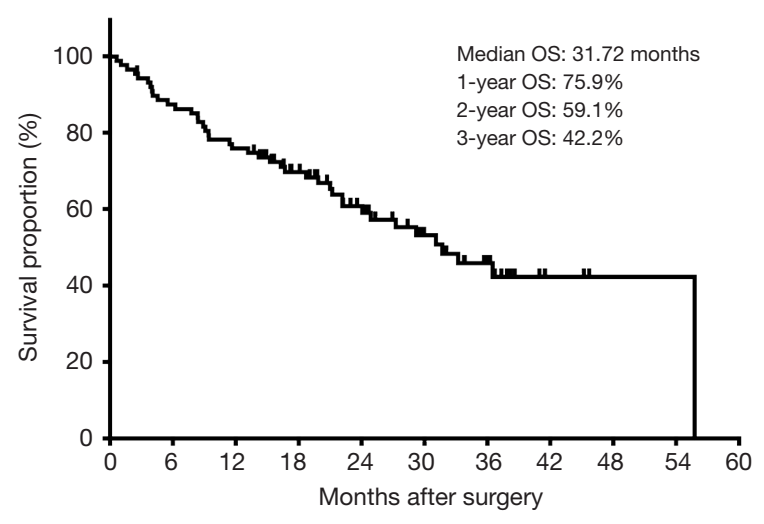

Figure 1 Overall survival of entire cohort.

metastases were shown in Table S5.

\section{Overall survival and subgroup analysis}

During a median follow-up of 20.87 months, 41 (46.6\%) patients died and 5 patients were lost to follow up. The median OS of entire cohort was 31.72 months (Figure 1). The estimated 1-, 2- and 3-year OS rates were 75.9\%, $59.1 \%$ and $42.2 \%$, respectively.

Univariate analysis showed age, sex, smoking history, clinical $\mathrm{T}$ stage, tumor histology, site of metastases, treatment for metastases and adjuvant treatment as the major factors that were significantly associated with OS of patients $(\mathrm{P}<0.05)$. However, no prognostic significance was observed with respect to CCI score, clinical $\mathrm{N}$ stage and extent of pulmonary resection (Table 3). After adjusting in multivariate analysis, only age, clinical $\mathrm{T}$ stage, site of metastases and adjuvant treatment remained statistically significant for prognostic prediction (Table 4).

Subgroup analysis showed 3 -year OS rates of $53.3 \%$ in patients younger than 60 years and $0 \%$ in those aged 60 years or older. Meanwhile, patients with clinical T1-2 NSCLC had significant prolonged survival when compared with those with clinical T3-4 disease (median: 55.78 vs. 19.88 months, $\mathrm{P}=0.003)$. The estimated 3-year OS rates were $52.73 \%$ and $23.85 \%$, respectively (Figure $2 A$ ). However, clinical N2-3 status showed no significant influence on survival time (24.08 vs. 31.71 months, $\mathrm{P}=0.197$, Figure $2 B$ ). Besides, patients with $1-3$ brain metastases showed better OS (median: 55.78 months), followed by those with pleural metastases, 1-3 extra-brain metastases and multiple metastases (36.51, 15.28 and 13.18 months, $\mathrm{P}=0.008)$. The corresponding 3 -year OS were $60.80 \%$,
49.01\%, 22.02\% and 33.33\% (Figure 3). Also, adjuvant therapies showed obvious effects on OS of patients. Patients with TKI-based therapy had obviously superior survival, and median OS was not reached during our follow-up. The corresponding data were 24.08 months in chemotherapy group, 3.607 months in no treatment group and 33.22 months in unknown group. The estimated 3-year OS rates of these 4 subgroups were $76.3 \%, 17.1 \%$, censored and $40.7 \%$, respectively $(\mathrm{P}<0.001)$ (Figure 4$)$.

\section{Discussion}

Studies exploring the effectiveness of surgical treatment had been reported during the past decades, though systematic treatment was recommended for stage IV NSCLC. Palliative or even curative intent surgery was more frequently applied in selected patients (11-16). In the present study, surgical outcome of stage IV NSCLC was analyzed in the context of multimodality therapy. The 1-, 2- and 3-year OS rates were $75.9 \%, 59.1 \%$ and $42.2 \%$, with the MOS of 31.72 months. These results seem acceptable and encouraging when compared with the previous ones $(7,8,10,17)$.

Up to now, there is no definite selection criteria of potential candidates of stage IV for surgical treatment. Thus, a single center analysis of our institution was summarized to contribute to the future studies. Some prognostic factors were found to correlated to survival, such as age, clinical $\mathrm{T}$ stage, metastatic sites and adjuvant treatment modality.

The characteristics which might predict the potential subgroup benefit from surgery have not been identified. The national analysis by Yang et al. indicated age, sex, race, CCI score, clinical $\mathrm{T}$ and $\mathrm{N}$ stage as independent prognostic factors (10). However, other studies suggested that smoking history, performance status, histology types and/or best $\mathrm{N}$ stage were independently associated with OS of surgical stage IV patients $(17,18)$. Meanwhile, still other studies identified no independent predictors in surgically resected stage IV patients $(8,19)$. In the present study, age, clinical $\mathrm{T}$ stage and site of metastasis were major characteristics that were independently associated with OS of patients. By contrast, factors including smoking status, CCI score, tumor histology, clinical $\mathrm{N}$ stage, extent of pulmonary resection and treatment for metastases were not found to be prognostic. The heterogeneity in study population and treatment modality make it really difficulty for inter-study reproducibility. 
Table 3 Univariate COX analysis of overall survival in surgical stage IV patients

\begin{tabular}{|c|c|c|c|}
\hline \multirow{2}{*}{ Variables } & \multicolumn{3}{|c|}{ Univariate analysis } \\
\hline & HR & $95 \% \mathrm{Cl}$ & $P$ value \\
\hline Age $\geq 60$ years (vs. <60) & 2.668 & $1.423-5.001$ & 0.001 \\
\hline Non-smoker (vs. smoker) & 0.398 & $0.212-0.747$ & 0.003 \\
\hline Non-SCC (vs. SCC) & 0.293 & $0.138-0.624$ & 0.001 \\
\hline cT 3-4 (vs. T1-2) & 2.478 & $1.331-4.614$ & 0.003 \\
\hline cN 2-3 (vs. N0-1) & 1.143 & $0.588-2.220$ & 0.197 \\
\hline $1-3$ brain metastases & 1.014 & $0.381-2.704$ & 0.977 \\
\hline $1-3$ metastases in 1 extra-brain organ & 2.959 & $1.390-6.297$ & 0.005 \\
\hline Multiple metastases & 2.721 & $1.015-7.293$ & 0.047 \\
\hline Pulmonary resection & & & 0.738 \\
\hline Lobectomy & Ref & 1 & \\
\hline Sublobar resection & 0.878 & $0.208-3.709$ & 0.859 \\
\hline Pneumonectomy & 1.280 & $0.653-2.508$ & 0.473 \\
\hline TKI or TKI + chemotherapy & 0.211 & $0.083-0.534$ & 0.001 \\
\hline No treatment & 4.822 & $2.032-11.443$ & $<0.001$ \\
\hline Unknown & 0.730 & $0.305-1.748$ & 0.480 \\
\hline
\end{tabular}

$\mathrm{HR}$, hazard ratio; $\mathrm{Cl}$, confidence interval; $\mathrm{CCl}$, Charlson comorbidity index; SCC, squamous cell carcinoma; TKI, tyrosine kinase inhibitor.

Lymph nodes status may be potentially influenced survival of stage IV NSCLC after surgery. Positive lymph nodes were found to be an important unfavorable survival in surgery treated patients with brain metastases $(20,21)$. However, no such association was observed in patients with other metastatic sites $(9,22,23)$. Majority of previous studies used pathological stage to perform Cox regression and evaluate risk factors. However, due to the pathological stage of primary tumor, especially $\mathrm{pN}$ stage, was difficult to be obtained in perioperative time, we choose clinical stage to do the analysis as it is more meaningful for general clinical practice. The present study indicated no prognostic significance of clinical N2/N3 status in surgery treated stage IV patients. In light of systematic disease of stage IV NSCLC and small sample size in present study, whether clinical $\mathrm{N}$ stage has a significant prognostic effect in surgically resected stage IV patients still need further investigation. 
Table 4 Multivariate COX analysis of overall survival in surgical stage IV patients

\begin{tabular}{|c|c|c|c|}
\hline Variables & \multicolumn{3}{|c|}{ Multivariate analysis } \\
\hline Age $\geq 60$ (vs. <60) & 2.302 & $1.154-4.593$ & 0.018 \\
\hline cT 3-4 (vs. cT1-2) & 2.969 & $1.396-6.313$ & 0.005 \\
\hline \multicolumn{4}{|l|}{ Sites of metastasis } \\
\hline $1-3$ brain metastases & 1.812 & $0.617-5.321$ & 0.279 \\
\hline 1-3 metastases in 1 extra-brain organ & 2.572 & $1.130-5.852$ & 0.024 \\
\hline Multiple metastases & 3.603 & $1.195-10.861$ & 0.023 \\
\hline \multicolumn{4}{|l|}{ Adjuvant treatment } \\
\hline No treatment & 5.781 & $2.135-15.650$ & 0.001 \\
\hline Unknown & 1.158 & $0.434-1.396$ & 0.770 \\
\hline
\end{tabular}

$\mathrm{HR}$, hazard ratio; $\mathrm{Cl}$, confidence interval; TKI, tyrosine kinase inhibitor.

There was study compared the physiologic impact between VATs and thoracotomy. VATs group would get more favorable outcomes on pain, pulmonary function and short-time recovery (24). However, we excluded the variable from COX regression at the final analysis of the present study, because we considered that multidisciplinary treatment modality, including local treatment for malignant lesions, systematic adjuvant therapy and general status of tumor were the key prognosis for advanced stage NSCLC. The analysis of surgical approach in COX regression might cause confounding bias.

Pleural dissemination or effusion was considered contraindication to surgical treatment for NSCLC. However, In Jin et al.'s opinion, remove of malignant lesion would alleviate tumor burden and recover the immune functions of NSCLC patients, even with pleural dissemination at diagnosed time. It would be benefit for further combined therapies (25). In a study conducted by Iida $e t$ al., $77.8 \%$ of patients diagnosed with pleural effusion or nodes before surgery underwent surgical resection for lung, and a 5 -year OS of $33.1 \%$ was noted in patients underwent surgery for NSCLC with pleural metastases (17). No survival difference was found between patients diagnosed before and at thoracotomy. The present study was consistent with these results. The patients with preoperative pleural effusion or nodes were with good performance status and controllable primary tumor. Radical surgery might be a choice for attenuating symptoms and further systematic therapies.

Metastatic sites are associated with survivals of surgery treated stage IV NSCLC. So far, NSCLC patients with pleural metastases and brain oligometastases seems benefit from the resection of primary lung tumors. Surgery or stereotactic radiotherapy (SRS) for brain metastatic NSCLC has been reported to associate with a 5-year OS rate of $11-36.8 \%(9,13,20,26,27)$. Moreover, a better OS was noted in patients with brain metastases when compared with those with metastases outside brain (median: 94.8 vs. 24.0 months), although no statistical significance was achieved (28). Meanwhile, patients with adrenal metastases are likely to benefit from lung surgery. Most of previous studies showed that both lung and adrenal resection could result in favorable outcomes with a 5 -year OS from $9.1 \%$ to $34 \%$ $(22,23,29,30)$. On the contrary, a recent study by Huang et al. indicated no survival advantage of surgical resection in patients with metastatic NSCLC to the adrenal (31). Furthermore, bone metastatic patients may get unfavorable survival compared with brain or adrenal. In the study conducted by Kawano and colleagues, a 5-year OS of only $14.3 \%$ was found in patients with bone metastases, while $30.5 \%$ of those with extra-bone metastases (8). In the present study, surgical resection for NSCLC with pleural 

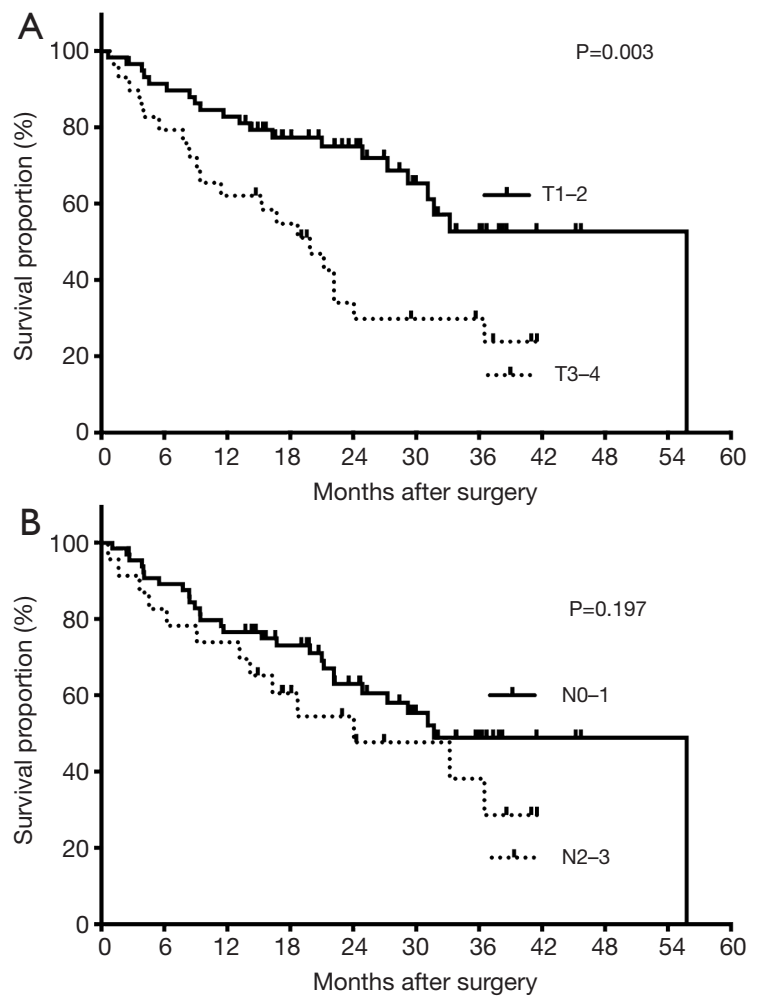

Figure 2 The Kaplan-Meier survival analysis based on clinical T stage (A) and clinical $\mathrm{N}$ stage (B).

metastases or 1-3 brain metastases resulted in better survival than those with 1-3 extra-brain metastases or multiple metastases. Our results confirmed previous findings in a way. Site and extent of metastasis should be therefore cautiously considered when selecting patients for surgical resection.

Up to now, brain surgery and SRS are both common ways to solve brain lesions. A series of studies have proved the equal effectiveness between brain surgery and SRS on treating brain lesion $(32,33)$. Brain surgery is more suitable for single metastases, especially when it is larger than $4 \mathrm{~cm}$, while SRS is more frequently used for multiple metastases. In the present study, there were 7 patients with single brain metastasis receiving brain surgery and 6 receiving SRS. Another 5 patients with 2 or 3 metastases were all treated with SRS. The results of present study showed favorable outcomes on treating brain metastatic patients with an MOS of 55.72 months.

Stereotactic body radiotherapy (SBRT) could be a considerable choice for extra-cranial metastases. It is a noninvasive approach to deal metastases with favorable local

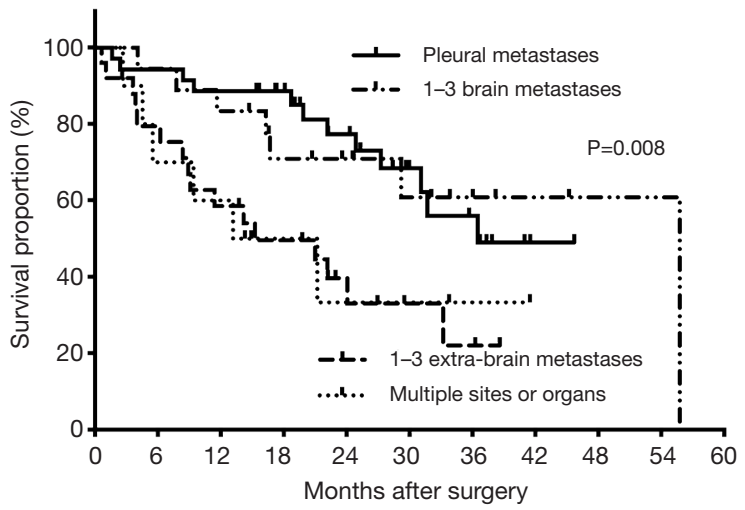

Figure 3 The Kaplan-Meier survival analysis based on site of metastases.

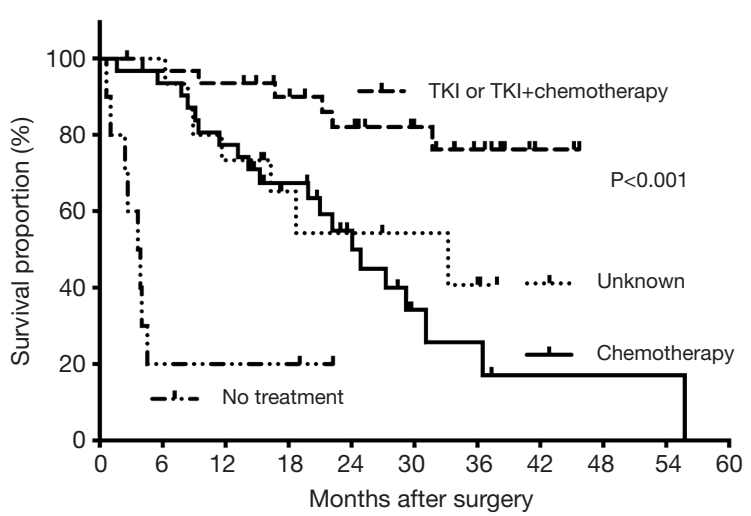

Figure 4 The Kaplan-Meier survival analysis based on adjuvant therapy. TKI: tyrosine kinase inhibitor.

control and low toxicity. In a study conducted by Toesca et al., the 3-year local control rate of SBRT for adrenal metastases exceeded $80 \%$ (34). For patients with vertebral metastases, SBRT is effective in controlling tumor growth, but also pain relief. Guckenberger et al. conducted a multicenter analysis of SBRT for vertebral metastases, local control of tumor progression was got in $84 \%$ of the patients and pain relief was achieved in $58 \%$ of the cohort (35).

The role of adjuvant treatment for surgical treated stage IV NSCLC was unclear. Most of previous studies showed that adjuvant therapy did not contribute to survival, especially in two prospective studies $(14,16)$. However, a significantly association was obtained in a national analysis of surgically treated stage IV NSCLC in America, under the condition of real-world analysis (10). Our study showed obvious survival benefit of chemotherapy compared with no adjuvant treatment. Sequential systematic therapy may 
eliminate the hematogenous or indolent micro-metastases.

The most important finding of the present study was the excellent survival of patients treated with adjuvant EGFR-TKI therapy after lung surgery. To our knowledge, ADJUVANT and EVAN studies have both proved the superiority of adjuvant EGFR-TKI therapy to prolong disease-free survival in stage II and IIIA NSCLC compared with cisplatin-based chemotherapy $(36,37)$, whereas the combination of surgery and adjuvant EGFR-TKI for advanced NSCLC has not been fully discussed before. Unsurprisingly but importantly, adjuvant EGFR-TKI therapy was associated with better survival when compared with chemotherapy in the present study. The MOS was not reached due to our not long enough follow-up time. Compared with previous studies, EGFR-TKI was widely used as standard care for patients enrolled in the present study (Jan 2014 to Jun 2017). However, this is a small sample size study with not long enough follow-up, as well as potential selection bias.

Meanwhile, the application of third generation EGFRTKI osimertinib for advanced NSCLC resulted in an estimated 3-year OS of $57.3 \%$ in FLAURA study (38). The 3 -year OS rate of patients treated with surgery combined EGFR-TKI was $76.3 \%$ in present study. Different demographic characteristics and baselines make the direct comparison of figures not reliable. However, it reminded us the combination of surgery and EGFR-TKI might contribute better survival to selected patients than EGFRTKI alone.

Nonetheless, in view of the favorable outcomes in the present study, the authors suggest that the following topics could be discussed in the future. First, the effectiveness of adjuvant EGFR-TKI therapy compared with adjuvant chemotherapy for surgical stage IV NSCLC as a standard adjuvant treatment. Second, whether surgery combined adjuvant EGFR-TKI has favorable influence on survival compared with EGFR-TKI alone for stage IV NSCLC. Third, whether "potential EGFR-TKI treated patient" could be adopted as one of the eligibilities for surgery selection in stage IV NSCLC.

This study also has several limitations. Primarily, this is a single-center study with small sample size and not long enough follow-up. It was difficult to calculate 5-year survival rate of entire cohort accurately, and a prediction of death risk was not reliable based on data acquired now. Besides, surgery was performed in a selection-biased population. Also, the heterogeneous nature of metastatic patients and multiplicity of treatment modality may further prevent us from definite conclusion. However, in view of the favorable outcomes observed in our study, there is still a chance for advanced NSCLC to get satisfied survival from surgery. Future studies are required to identify potential candidates for surgical resection in the context of personalized medicine.

In conclusion, we retrospectively analyzed characteristics and outcomes of stage IV NSCLC who underwent multimodality therapy including surgery for lung. A 3-year OS of $42 \%$ were achieved. Patients with younger age, lower clinical $\mathrm{T}$ stage and pleural metastases or 1-3 brain metastases were more likely to benefit. Adjuvant EGFRTKI therapy was associated with significantly better survival of surgical patients when compared with chemotherapy. Lung surgery may be a considerable choice for stage IV NSCLC in context of multimodality therapy.

\section{Acknowledgments}

We would like to thank Haijue Wang, Xiaoxiang Jie, Jinyun Sun, Xuefeng Li and Kang Hu (LinkDoc Inc. Beijing, China) and assistant professor Linna Liu from Tangdu hospital for their valuable discussion.

Funding: This study was supported by the National Natural Science Foundation (No. 81572252 and No.81001041) and the Natural Science Foundation of Shaanxi Province (No. 2016JM8087).

\section{Footnote}

Conflicts of Interest: The authors have no conflicts of interest to declare.

Ethical Statement: The authors are accountable for all aspects of the work in ensuring that questions related to the accuracy or integrity of any part of the work are appropriately investigated and resolved.

\section{References}

1. Bray F, Ferlay J, Soerjomataram I, et al. Global cancer statistics 2018: GLOBOCAN estimates of incidence and mortality worldwide for 36 cancers in 185 countries. CA Cancer J Clin 2018;68:394-424.

2. Pereira JL, Gomes M, Teixeira AL, et al. Potential and importance of metalloproteinases and interleukins in inflammation and metastasization in non-small cell lung cancer. Transl Cancer Res 2018;7:782-95. 
3. Socinski MA, Crowell R, Hensing TE, et al. Treatment of non-small cell lung cancer, stage IV: ACCP evidencebased clinical practice guidelines ( $2 \mathrm{nd}$ edition). Chest 2007;132:277s-89s.

4. Non-Small Cell Lung Cancer Collaborative Group. Chemotherapy and supportive care versus supportive care alone for advanced non-small cell lung cancer. Cochrane Database Syst Rev 2010;(5):CD007309.

5. Yang JC, Wu YL, Schuler M, et al. Afatinib versus cisplatin-based chemotherapy for EGFR mutation-positive lung adenocarcinoma (LUX-Lung 3 and LUX-Lung 6): analysis of overall survival data from two randomised, phase 3 trials. Lancet Oncol 2015;16:141-51.

6. Seto T, Kiura K, Nishio M, et al. CH5424802 (RO5424802) for patients with ALK-rearranged advanced non-small-cell lung cancer (AF-001JP study): a single-arm, open-label, phase 1-2 study. Lancet Oncol 2013;14:590-8.

7. Hanagiri T, Takenaka M, Oka S, et al. Results of a surgical resection for patients with stage IV non--small-cell lung cancer. Clin Lung Cancer 2012;13:220-4.

8. Kawano D, Takeo S, Katsura M, et al. Surgical treatment of stage IV non-small cell lung cancer. Interact Cardiovasc Thorac Surg 2012;14:167-70.

9. Collaud S, Stahel R, Inci I, et al. Survival of patients treated surgically for synchronous single-organ metastatic NSCLC and advanced pathologic TN stage. Lung Cancer 2012;78:234-8.

10. Yang CJ, Gu L, Shah SA, et al. Long-term outcomes of surgical resection for stage IV non-small-cell lung cancer: A national analysis. Lung Cancer 2018;115:75-83.

11. Torre M, Quaini E, Chiesa G, et al. Synchronous brain metastasis from lung cancer. Result of surgical treatment in combined resection. J Thorac Cardiovasc Surg 1988;95:994-7.

12. Luketich JD, Burt ME. Does resection of adrenal metastases from non-small cell lung cancer improve survival? Ann Thorac Surg 1996;62:1614-6.

13. Bonnette P, Puyo P, Gabriel C, et al. Surgical management of non-small cell lung cancer with synchronous brain metastases. Chest 2001;119:1469-75.

14. Downey RJ, Ng KK, Kris MG, et al. A phase II trial of chemotherapy and surgery for non-small cell lung cancer patients with a synchronous solitary metastasis. Lung Cancer 2002;38:193-7.

15. Congedo MT, Cesario A, Lococo F, et al. Surgery for oligometastatic non-small cell lung cancer: Longterm results from a single center experience. J Thorac Cardiovasc Surg 2012;144:444-52.
16. Endo C, Hasumi T, Matsumura Y, et al. A Prospective Study of Surgical Procedures for Patients With Oligometastatic Non-Small Cell Lung Cancer. Ann Thorac Surg 2014;98:258-64.

17. Iida T, Shiba M, Yoshino I, et al. Surgical Intervention for Non-Small-Cell Lung Cancer Patients with Pleural Carcinomatosis: Results From the Japanese Lung Cancer Registry in 2004. J Thorac Oncol 2015;10:1076-82.

18. Liu T, Liu H, Wang G, et al. Survival of M1a Non-Small Cell Lung Cancer Treated Surgically: A Retrospective Single-Center Study. Thorac Cardiovasc Surg 2015;63:577-82.

19. Mordant P, Arame A, Foucault C, et al. Surgery for metastatic pleural extension of non-small-cell lung cancer. Eur J Cardiothorac Surg 2011;40:1444-9.

20. Billing PS, Miller DL, Allen MS, et al. Surgical treatment of primary lung cancer with synchronous brain metastases. J Thorac Cardiovasc Surg. 2001;122:548-53.

21. Granone P, Margaritora S, D'Andrilli A, et al. Nonsmall cell lung cancer with single brain metastasis: the role of surgical treatment. Eur J Cardiothorac Surg 2001;20:361-6.

22. Porte H, Siat J, Guibert B, et al. Resection of adrenal metastases from non-small cell lung cancer: a multicenter study. Ann Thorac Surg 2001;71:981-5.

23. Mercier O, Fadel E, de Perrot M, et al. Surgical treatment of solitary adrenal metastasis from non-small cell lung cancer. J Thorac Cardiovasc Surg 2005;130:136-40.

24. Pu Q, Ma L, Mei J, et al. Video-assisted thoracoscopic surgery versus posterolateral thoracotomy lobectomy: A more patient-friendly approach on postoperative pain, pulmonary function and shoulder function. Thorac Cancer 2013;4:84-9.

25. Jin WB, Liang CY, Peng YH, et al. Pleuropneumonectomy for diffuse pleural metastasis in primary lung cancer. J Cancer Res Ther 2013;9:S92-7.

26. Mussi A, Pistolesi M, Lucchi M, et al. Resection of single brain metastasis in non-small-cell lung cancer: prognostic factors. J Thorac Cardiovasc Surg 1996;112:146-53.

27. Furak J, Trojan I, Szoke T, et al. Lung cancer and its operable brain metastasis: survival rate and staging problems. Ann Thorac Surg 2005;79:241-7.

28. Yamaguchi M, Edagawa M, Suzuki Y, et al. Pulmonary Resection for Synchronous M1b-cStage IV NonSmall Cell Lung Cancer Patients. Ann Thorac Surg 2017;103:1594-9.

29. Raz DJ, Lanuti M, Gaissert HC, et al. Outcomes of Patients With Isolated Adrenal Metastasis From 
Non-Small Cell Lung Carcinoma. Ann Thorac Surg 2011;92:1788-92.

30. Pfannschmidt J, Schlolaut B, Muley T, et al. Adrenalectomy for solitary adrenal metastases from nonsmall cell lung cancer. Lung Cancer 2005;49:203-7.

31. Huang SH, Kong QL, Chen XX, et al. Adrenalectomy does not improve survival rates of patients with solitary adrenal metastasis from non-small cell lung cancer. Ther Clin Risk Manag 2017;13:355-60.

32. Aoyama H, Shirato H, Tago M, et al. Stereotactic radiosurgery plus whole-brain radiation therapy vs stereotactic radiosurgery alone for treatment of brain metastases: a randomized controlled trial. JAMA 2006;295:2483-91.

33. Lin X, DeAngelis LM. Treatment of Brain Metastases. J Clin Oncol 2015;33:3475-84.

34. Toesca DAS, Koong AJ, von Eyben R, et al. Stereotactic body radiation therapy for adrenal gland metastases: Outcomes and toxicity. Adv Radiat Oncol 2018;3:621-9.

35. Guckenberger M, Mantel F, Gerszten PC, et al. Safety and efficacy of stereotactic body radiotherapy as primary

Cite this article as: Zhang $\mathrm{C}$, Wang $\mathrm{L}, \mathrm{Li} W$, Huang $\mathrm{Z}$, Liu W, Bao P, Lai Y, Han Y, Li X, Zhao J. Surgical outcomes of stage IV non-small cell lung cancer: a single-center experience. J Thorac Dis 2019;11(12):5463-5473. doi: $10.21037 /$ jtd.2019.11.30 treatment for vertebral metastases: a multi-institutional analysis. Radiat Oncol 2014;9:226.

36. Yue $\mathrm{D}, \mathrm{Xu} \mathrm{S}$, Wang Q, et al. Erlotinib versus vinorelbine plus cisplatin as adjuvant therapy in Chinese patients with stage IIIA EGFR mutation-positive non-small-cell lung cancer (EVAN): a randomised, open-label, phase 2 trial. Lancet Respir Med 2018;6:863-73.

37. Zhong WZ, Wang Q, Mao WM, et al. Gefitinib versus vinorelbine plus cisplatin as adjuvant treatment for stage II-IIIA (N1-N2) EGFR-mutant NSCLC (ADJUVANT/ CTONG1104): a randomised, open-label, phase 3 study. Lancet Oncol 2018;19:139-48.

38. Dyer M, Green M, Jones S, et al. Estimating longterm survival of previously untreated patients with EGFR mutation-positive (EGFRm) advanced non-small cell lung cancer (NSCLC) who received osimertinib in the FLAURA study. 2019 ASCO Annual Meeting. 2019. Available online: http://abstracts.asco.org/239/ AbstView_239_254741.html 
Supplementary

Table S1 Detailed information and treatment of pleural metastases

\begin{tabular}{|c|c|c|}
\hline Sites & Interventions & Total $(n=35)$ \\
\hline Diagnosed at preoperative time & & $14(40 \%)$ \\
\hline Malignant pleural effusion & Decortication $^{\dagger}$ & 4 \\
\hline Malignant pleural effusion & Radio knife burning ${ }^{\ddagger}$ & 1 \\
\hline Pleural nodes & Resection $^{\dagger}$ & 6 \\
\hline Pleural dissemination & No treatment & 3 \\
\hline Diagnosed during operations & & $21(60 \%)$ \\
\hline Pleural dissemination & Decortication $^{\dagger}$ & 1 \\
\hline Pleural dissemination & No treatment & 5 \\
\hline Pleural nodes & Resection $^{\dagger}$ & 12 \\
\hline Pleural nodes & Radio knife burning ${ }^{\ddagger}$ & 3 \\
\hline
\end{tabular}

${ }^{\dagger}$, curative local treatment; ${ }^{\ddagger}$, partial treatment.

Table S2 Detailed information and treatment of brain metastases

\begin{tabular}{lc}
\hline Metastases and intervention & Total $(\mathrm{n}=18)$ \\
\hline 1 metastasis $^{\prime}$ & $13(72.2 \%)$ \\
Surgery $^{\dagger}$ & 5 \\
$\mathrm{SRS}^{\dagger}$ & 6 \\
Surgery + WBRT $^{\dagger}$ & 1 \\
Surgery + SRS & \\
2 metastases $^{\dagger}$ & 1 \\
SRS $^{\dagger}$ & $2(11.1 \%)$ \\
3 metastases & 2 \\
SRS $^{\dagger}$ & $3(16.7 \%)$ \\
\hline
\end{tabular}

${ }^{\dagger}$, curative local treatment. SRS, stereotactic radiotherapy; WBRT, whole brain radiotherapy. 
Table S3 Detailed information and treatment of 1 to 3 extra-brain metastases

\begin{tabular}{|c|c|c|c|}
\hline Sites & Metastases & Intervention & Total $(n=25)$ \\
\hline Contralateral lung & 1 & No treatment & $3(12.0 \%)$ \\
\hline Adrenal & & & $10(40.0 \%)$ \\
\hline Ipsilateral & 1 & $\mathrm{SRS}^{\dagger}$ & 4 \\
\hline Ipsilateral & 1 & No treatment & 2 \\
\hline Contralateral & 1 & $\mathrm{SRS}^{\dagger}$ & 2 \\
\hline Contralateral & 1 & No treatment & 2 \\
\hline Bone & & & $11(44.0 \%)$ \\
\hline TV & 1 & $\mathrm{SRS}^{\dagger}$ & 2 \\
\hline TV & 2 & No treatment & 1 \\
\hline Lumbar & 1 & Bone cement ${ }^{\ddagger}$ & 1 \\
\hline$C V+T V$ & 3 & No treatment & 1 \\
\hline Rib & 2 & En-bloc resection ${ }^{\dagger}$ & 1 \\
\hline Rib & 1 & No treatment & 1 \\
\hline Rib + TV & 2 & Rib resection ${ }^{\ddagger}$ & 1 \\
\hline Rib + TV & 2 & No treatment & 1 \\
\hline Sternum + hip & 1 & No treatment & 1 \\
\hline Thigh & 1 & No treatment & 1 \\
\hline Liver & 1 & $\mathrm{RA}^{\dagger}$ & $1(4.0 \%)$ \\
\hline
\end{tabular}

${ }^{\dagger}$, curative local treatment; ${ }^{\ddagger}$, partial treatment. TV, thoracic vertebrae; CV, cervical vertebrae; SRS, stereotactic radiotherapy; RA, radiofrequency ablation.

Table S4 Detailed information and treatment of multiple metastases

\begin{tabular}{|c|c|c|}
\hline Sites & Intervention & Total $(n=10)$ \\
\hline With pleural involvement & & $5(50 \%)$ \\
\hline \multicolumn{3}{|c|}{ Pleural diagnosed at preoperative time } \\
\hline Pleural + brain & Node resection + brain $\mathrm{SRS}^{\dagger}$ & 1 \\
\hline Pleural + rib & Decortication + rib resection $^{\dagger}$ & 1 \\
\hline Pleural + contralateral lung & Node resection + no treatment ${ }^{\ddagger}$ & 1 \\
\hline \multicolumn{3}{|c|}{ Pleural diagnosed during operation } \\
\hline Pleural + brain & Radio knife burning + brain $\mathrm{SRS}^{\ddagger}$ & 1 \\
\hline Pleural + contralateral lung & Radio knife burning + no treatment ${ }^{\ddagger}$ & 1 \\
\hline Without pleural involvement & & $5(50 \%)$ \\
\hline Bone (5 sites) & No treatment & 1 \\
\hline Brain + adrenal & No treatment & 1 \\
\hline Bone + adrenal & No treatment & 1 \\
\hline Bone + contralateral lung & No treatment & 2 \\
\hline
\end{tabular}

${ }^{\dagger}$, curative local treatment; ${ }^{\ddagger}$, partial treatment. SRS, stereotactic radiotherapy.

Table S5 Detailed information of adjuvant EGFR-TKI for different metastases

\begin{tabular}{lc} 
Sites & Patients received EGFR-TKI/total patients (\%) \\
\hline Pleural dissemination & $15 / 35(42.86)$ \\
$1-3$ brain metastases & $9 / 18(50.0)$ \\
$1-3$ extra-brain metastases & $3 / 25(12.0)$ \\
Multiple metastases or organs & $4 / 10(40.0)$
\end{tabular}

EGFR-TKI, epidermal growth factor receptor-tyrosine kinase inhibitor. 